\title{
=ALTERIDAD Aprendizaje-servicio en la enseñanza de la Sociología a futuros docentes
}

\section{Service-Learning in the teaching of Sociology for future teachers}

Dra. Paloma Candela-Soto es docente e investigadora de la Universidad de Castilla-La Mancha (España) (Paloma.Candela@ uclm.es) (https://orcid.org/0000-0002-4857-8747)

(D) Dra. María Carmen Sánchez-Pérez es docente titular e investigadora de la Universidad de Castilla-La Mancha (España) (Mariacarmen.Sanchez@uclm.es) (https://orcid.org/0000-0002-7195-7446)

(D) Dra. Mercedes Ávila-Francés es docente e investigadora de la Universidad de Castilla-La Mancha (España) (Mercedes.Avila@ uclm.es) (https://orcid.org/0000-0003-4253-4345)

Recibido: 2020-07-11 / Revisado:: 2020-12-07 / Aceptado: 2020-12-09 / Publicado: 2021-01-01

\section{Resumen}

Este artículo reflexiona y hace balance de las contribuciones del aprendizaje-servicio (ApS) a la docencia universitaria y, en concreto, a la formación inicial del profesorado. A partir de la evaluación de un proyecto de innovación docente llevado a cabo en las Facultades de Educación de la Universidad de Castilla La Mancha (UCLM), se profundiza en la percepción de los estudiantes sobre la utilidad del ApS en el aprendizaje académicocompetencial y en las experiencias vividas en escenarios escolares reales donde han observado y aplicado sus aprendizajes teóricos. La valoración de la adquisición de competencias concretas del Grado, la disposición y desempeño para comprender problemas sociales que deberán afrontar en su futuro profesional, así como los sentimientos y emociones experimentadas, son algunos de los objetivos que guían nuestro trabajo. Se trata de un estudio empírico de metodología mixta, mediante un cuestionario online y el análisis de las narraciones reflexivas de los estudiantes con una muestra de 167 alumnos y alumnas. Los resultados revelan la elevada satisfacción del alumnado participante, especialmente en variables vinculadas con la adquisición de competencias profesionales y personales, destacando las destrezas críticas y reflexivas que favorecen el aprendizaje significativo de la Sociología. Con todo, la investigación corrobora el carácter renovador del ApS en la enseñanza superior, una metodología que se adapta con idoneidad al desempeño competencial y formativo de las futuras maestras y maestros.

Descriptores: Aprendizaje-Servicio, Sociología de la Educación, innovación docente, competencias profesionales, percepciones de los estudiantes, responsabilidad social universitaria.

\section{Abstract}

This paper reflects and gives an account of the Service-Learning (S-L) contributions to university teaching and, more specifically, to the initial teacher training. It is part of an innovation project carried out in the Faculties of Education in the University of Castilla-La Mancha (UCLM). It deepens into students' perceptions about $S-L$ benefits for academic and skill learning. It also studies students' experiences at schools, where they were able to observe and apply their theoretical learning. Some of the objectives guiding this study are to assess the Degree skills acquisition, their ability to understand social problems that they will have to face in their professional future, as well as the feelings and emotions experienced. This empirical mixed methodology study (by an online questionnaire and student narratives analysis) reaches a 167 students sample. Results verify their high level of satisfaction with the S-L project, especially in those variables linked to the professional and personal skills, highlighting the critical and reflective skills that promote a significant learning in Sociology. All in all, this study verifies the renewing power of $S-L$ in higher education, as this methodology effectively adapts to future teachers' competence and training performance.

Keywords: Service-Learning, Sociology of Education, teaching innovation, professional skills, students' perceptions, university social responsibility.

Forma sugerida de citar: Candela-Soto, P., Sánchez-Pérez, M.C., \& Ávila-Francés-M. (2021). Aprendizaje-servicio en la enseñanza de la Sociología a futuros docentes. Alteridad, 16(1), 38-50. https://doi.org/10.17163/alt.v16n1.2021.03 


\section{Introducción}

Este artículo parte de los resultados obtenidos en el proyecto de innovación docente desarrollado por las Facultades de Educación de la Universidad de Castilla-La Mancha (UCLM), orientado a implantar la metodología de AprendizajeServicio en la formación de los futuros docentes. Con un marcado carácter interdisciplinar, ha estado liderado desde sus comienzos (en el curso académico 2017-18) por profesoras del Área de Sociología en los Grados de Educación Infantil y Primaria. El proyecto buscaba dotar a las asignaturas de mayor contenido práctico, ofreciendo al alumnado la posibilidad de participar en proyectos altruistas en contextos de aprendizaje reales, que contribuyesen a la trasformación social y al bienestar de la comunidad. Al tiempo, perseguía promover una relación ética y moral de los estudiantes con el entorno. Este proyecto de aprendizaje-servicio (en adelante ApS) se fundamenta en la esencial relevancia que tienen en la formación de futuros y futuras profesionales de la educación competencias como: la innovación docente, la responsabilidad social universitaria, la calidad académica y la ética (Pérez-Gómez, 1993). A lo largo de los tres años de recorrido del proyecto, en el que se han implicado más de trescientos estudiantes de primer y segundo curso de los Grados de Educación y veinte profesores y profesoras, se ha podido constatar la motivación y el sentido del aprendizaje que representa el ApS, acercando a futuros maestros y maestras a escenarios reales donde observar y aplicar los aprendizajes teóricos de las asignaturas implicadas.

Desde la Sociología, más específicamente, los objetivos se orientaron a identificar situaciones de desigualdad en el contexto escolar y reflexionar sobre ellas; analizar el impacto que la educación tiene en la sociedad y viceversa e identificar los procesos sociales que están influyendo. Todo ello desde un aprendizaje de doble flujo: académico y vivencial. De ahí que la adquisición de competencias y contenidos de Sociología, así como el desarrollo de destrezas asociadas a los fundamentos del pensamiento y la acción sociológica centren el interés de nuestro estudio.

La metodología ApS, tras un proceso de desarrollo y consolidación (Tapia, 2008), llega a nuestros días con una relativa institucionalización, como demuestran tanto las numerosas redes nacionales e internacionales, extendidas en los distintos continentes, como los congresos, eventos y publicaciones específicas. Furco (2004) indica la existencia de más de doscientas definiciones diferentes de ApS publicadas tan solo en inglés y Tapia (2014) identifica como ApS aquellas prácticas que reúnen simultáneamente al menos tres de las siguientes características: la prestación de un servicio solidario a la comunidad, el protagonismo de los propios estudiantes en la ejecución del proyecto, una clara articulación de las actividades solidarias con el aprendizaje y la aplicación de saberes y competencias curriculares explícitas.

De este modo, el potencial renovador del ApS ha empezado a dejar huella también en la docencia universitaria, dotándola de un modelo educativo que combina la formación de profesionales de calidad y de ciudadanos activos, capaces de contribuir a la mejora y transformación de la sociedad. A su apuesta por una formación académica de calidad, que vaya más allá de aprender contenidos y desarrollar competencias, incorpora la preocupación por formar al estudiante en un contexto social y real, capacitándolo para analizar el impacto social, ético y ciudadano que su ejercicio comporta (Rubio \& Escofet, 2017, p. 20; Gutiérrez \& Moreno, 2018). Así mismo, se plantea como una metodología idónea para trabajar las competencias (Ferrán-Zubillaga \& Guinot-Viciano, 2012).

El trabajo que aquí se presenta focaliza su atención en dos aspectos esenciales: la contribución de la Sociología a la formación inicial del profesorado y su interrelación con el ApS. Se trata de una aportación de gran originalidad y relevancia, pues son escasas las aportaciones en este ámbito.

El objetivo general ha sido conocer la percepción de los estudiantes del Grado en Maestro 
de la UCLM sobre las contribuciones del ApS a su formación inicial docente, partiendo de las competencias y aprendizajes profesionales que maestras y maestros deberán enfrentar en el futuro. Más concretamente, se investigaron los siguientes ámbitos: (1) su percepción sobre la aportación de la metodología ApS al aprendizaje de la Sociología de la Educación; (2) en qué medida percibían que las destrezas que trabaja la metodología ApS pueden favorecer la adquisición de competencias del Grado de Maestro, (en concreto, las relativas a trabajo en equipo y el desarrollo de habilidades personales y profesionales); (3) los sentimientos asociados a su experiencia ApS y (4) la satisfacción general de los estudiantes con el proyecto ApS.

Si bien la Sociología tiene una presencia periférica en los planes de estudio de los actuales Grados de Educación, son muchos los análisis y especialistas que destacan su valiosa aportación a la formación docente (McNamara,1972; Daine \& Foster, 1976, en Loubet-Orozco, 2018, p. 9; Perrenoud, 2002; Guerrero-Serón, 2007; Venegas, 2012). Las voces que ayer (Dewey) y hoy defienden la pertinencia de la Sociología y, en particular de la Sociología de la Educación, coinciden en la actitud crítica y reflexiva que esta infunde en el alumnado de magisterio. Guerrero Serón (2007) llegó a plantear la formación del profesorado como destino natural de la Sociología de la Educación, un saber imprescindible que dota al alumnado "de los instrumentos teóricos y de análisis que le permitan comprender y actuar sobre el contexto social donde se están formando y donde van a desarrollar su trabajo" (p. 10).

El propósito principal de las asignaturas de Sociología es comprender la interrelación escuela-sociedad, prestando especial atención a las variables básicas de la desigualdad social: clase, género y etnia, como se demanda en la normativa que regula los planes de estudio de maestro. Desde este propósito, la "imaginación sociológica" (Mills, 1959) es considerada una cualidad esencial. A ella tenemos acceso todas las personas, permitiéndonos tomar conciencia de que nuestra manera de pensar y actuar indivi- dualmente está condicionada por la posición que ocupamos en la sociedad (Obiol i Francés et al., 2019 , p. 1086). En efecto, se trata - como plantean estos autores- de una competencia básica en la formación de futuras maestras y maestros, en la medida en que contribuye a formar docentes reflexivos y críticos.

En los Grados de Maestro, las asignaturas de Sociología suelen ubicarse en los primeros cursos, como parte de la formación básica, enmarcándose en un módulo de competencias denominado "Sociedad, familia y escuela". En los planes de estudios de la UCLM, este módulo se concreta en dos asignaturas de seis créditos cada una, denominadas "Sociología de la Educación" y "Educación y Sociedad", impartidas en primer y segundo curso respectivamente. Destacamos las siguientes competencias relacionadas con esta materia: 1) relacionar la educación con el medio; 2) analizar e incorporar de forma crítica las cuestiones más relevantes de la sociedad actual que afectan a la educación familiar y escolar, destacando el impacto social y educativo de los lenguajes audiovisuales y de las pantallas, los cambios en las relaciones de género e intergeneracionales, la multiculturalidad e interculturalidad, la discriminación e inclusión social y el desarrollo sostenible; 3) conocer la evolución histórica de la familia, los diferentes tipos de familias, de estilos de vida y educación en el contexto familiar.

Las competencias que se fijan para los títulos de Grado de Maestro emanan de diversas normativas. El Real Decreto de 2007, que regula la ordenación de las enseñanzas universitarias oficiales, establece que:

La formación en cualquier actividad profesional debe contribuir al conocimiento y desarrollo de los Derechos Humanos, los principios democráticos, de igualdad entre mujeres y hombres, de solidaridad, de protección medioambiental, de accesibilidad universal y de fomento de la cultura de la paz. (art. 3)

La Orden ECI/3857/2007 del 27 de diciembre, por la que se establecen los requisitos para la 
verificación de los títulos universitarios oficiales que habiliten para el ejercicio de la profesión de Maestro en Educación Primaria, plantea la necesidad de "regular espacios de aprendizaje respetuosos con los Derechos Humanos, colaborar con la comunidad educativa para fomentar la educación para una ciudadanía activa y fomentar la responsabilidad hacia un futuro sostenible" (art. 3). Para Educación Infantil, la Orden ECI/3854/2007 también incluye, entre otros aspectos, el "desarrollo de la competencia orientada a la reflexión en grupo, aceptación de normas y respeto a los demás" (art. 3).
El ApS, al conjugar en los estudiantes el aprendizaje académico con la prestación de un servicio a la comunidad orientado a su transformación y mejora, constituye una metodología ideal para desarrollar las competencias anteriores. Pero ¿en qué medida la participación en los proyectos ApS favorece el desarrollo de las competencias del Grado. La tabla 1, pone de manifiesto la intensa relación que existe entre las competencias de los títulos de Grado de Maestro y, más específicamente, de la materia de Sociología de la Educación, con la metodología ApS en Educación Primaria.

Tabla 1. Competencias de los títulos de Grado de Maestro relacionadas con el ApS y la materia de Sociología

\begin{tabular}{|c|c|c|}
\hline $\begin{array}{l}\text { Principios que deben inspirar los } \\
\text { títulos universitarios. } \\
\text { RD } 1393 / 2007 \text {, art. } 3.5\end{array}$ & $\begin{array}{c}\text { Competencias generales que los } \\
\text { estudiantes deben adquirir } \\
\text { (relacionadas con el ApS). } \\
\text { Orden ECI/3857/2007, Anexo, } \\
\text { Apartado 3 }\end{array}$ & $\begin{array}{c}\text { Competencias del módulo } \\
\text { Sociedad, familia y escuela } \\
\text { (relacionadas con la Sociología } \\
\text { y el proyecto ApS). } \\
\text { Orden ECI/3857/2007, Anexo, Apar- } \\
\text { tado } 5\end{array}$ \\
\hline $\begin{array}{l}\text { Respetar los derechos fundamen- } \\
\text { tales y de igualdad entre hombres } \\
\text { y mujeres. } \\
\text { - Promover los Derechos Humanos } \\
\text { y los principios de accesibilidad } \\
\text { universal. } \\
\text { - Contribuir a los valores propios de } \\
\text { una cultura de paz y de valores } \\
\text { democráticos. }\end{array}$ & $\begin{array}{l}\text { Diseñar y regular espacios de } \\
\text { aprendizaje en contextos de di- } \\
\text { versidad y que atiendan a la igual- } \\
\text { dad de género, a la equidad y al } \\
\text { respeto a los derechos humanos } \\
\text { que conforman los valores de la } \\
\text { formación ciudadana. } \\
\text { - Colaborar con los distintos sec- } \\
\text { tores de la comunidad educativa } \\
\text { y del entorno social. Asumir la di- } \\
\text { mensión educadora de la función } \\
\text { docente y fomentar la educación } \\
\text { democrática para una ciudadanía } \\
\text { activa. } \\
\text { Valorar la responsabilidad indivi- } \\
\text { dual y colectiva en la consecución } \\
\text { de un futuro sostenible. }\end{array}$ & $\begin{array}{l}\text { - Relacionar la educación con el medio } \\
\text { - Cooperar con las familias y la } \\
\text { comunidad. } \\
\text { - Analizar e incorporar de forma críti- } \\
\text { ca las cuestiones más relevantes de } \\
\text { la sociedad actual que afectan a la } \\
\text { educación familiar y escolar: impacto } \\
\text { social y educativo de los lenguajes } \\
\text { audiovisuales y de las pantallas; cam- } \\
\text { bios en las relaciones de género e } \\
\text { intergeneracionales; multiculturalidad } \\
\text { e interculturalidad; discriminación e } \\
\text { inclusión social y desarrollo sostenible }\end{array}$ \\
\hline
\end{tabular}

Fuente: Elaboración propia a partir de la ORDEN ECI/3857/2007, del 27 de diciembre y el Real Decreto 1393/2007, del 29 de octubre.

Nuestro desempeño formativo desde la Sociología persigue situar al alumnado en los procesos educativos y ante los retos que deberá enfrentar en su futuro profesional (Trottier \& Lessard, 2002, citados en Venegas, 2012, p. 407). Este propósito se pone a prueba, en particular, en la asignatura Educación y Sociedad, impartida en el segundo curso de las titulaciones estudiadas. En su desarrollo curricular, la materia de Sociología introduce a los estudiantes en los principios básicos de la investigación social, familiarizándolos con los principios metodológicos, enfoques, categorías y técnicas más usuales en la indagación educativa. En conexión con este 
aprendizaje exploratorio, se recorren cuestiones sociales de actualidad relacionadas con el ámbito educativo y familiar, se presentan ejemplos de estudios concretos, buscando mostrar a los estudiantes el equilibrio entre la investigación y su aplicación profesional. De ahí que los proyectos ApS desarrollados comprendiesen una gran diversidad de actuaciones, orientadas a dar respuesta a las demandas planteadas por los colegios colaboradores: apoyo a la lectoescritura en castellano e inglés, dinamización de recreos, patios inclusivos, grupos interactivos, promoción de la igualdad, apoyo escolar, educación saludable, dinamización de la biblioteca, cuentacuentos, etc.

De este modo, un frente de aprendizaje para los y las estudiantes que han participado en experiencias de ApS, ha sido el descubrimiento (o la toma de conciencia) del peso de las creencias, prejuicios o preconcepciones que, como docentes o educadores, construimos en torno al alumnado con el que trabajamos. Enfrentarse en la práctica con la meritocracia del pensamiento docente y sus implicaciones como predictores del rendimiento académico, es un aprendizaje necesario para desenmascarar la construcción social de estos juicios o representaciones que, como sabemos, el profesorado empieza a interiorizar en la formación inicial (Tarabini, 2015; San Román et al., 2015; López-Gamboa, 2019; Kaplan, 2012; Brozmanová et al., 2016). De ahí su potencial en la formación del profesorado universitario (Álvarez-Castillo et al., 2017).

Es precisamente en este contexto real ofrecido por el ApS donde se sitúa el significado de los conceptos, ideas y teorías que permiten avanzar en la formación del pensamiento práctico del estudiante, "donde ellos (y ellas) construyen recursos de comprensión y actuación como aprendices" (Pérez-Gómez, 2010, p. 48). Más adelante lo confirmaremos, con los resultados más relevantes de la exploración cualitativa realizada, tal y como es expresado por sus protagonistas: los propios estudiantes.

\section{Metodología}

El estudio se ha desarrollado desde un enfoque mixto, al combinar la recogida de datos cuantitativos (a través de cuestionarios online) y cualitativos (mediante las narraciones de experiencias en las memorias presentadas por los estudiantes para la evaluación de la asignatura). La orientación es, por tanto, principalmente descriptiva.

La muestra está configurada por el alumnado universitario de las Facultades de Educación de la UCLM, que cursó las asignaturas de Sociología de la Educación en primer curso y Educación y Sociedad en segundo, del Grado de Maestro en Educación Primaria, en los cursos académicos 2018/2019 y 2019/2020.

Los cuestionarios se dirigieron a estudiantes de primer curso, mientras que las memorias reflexivas se plantearon al alumnado de segundo, con más recorrido en la materia de Sociología.

En relación con el cuestionario, se invitó a participar a todos los estudiantes implicados en el proyecto ApS a través de la plataforma Moodle de las asignaturas (Campus Virtual UCLM), alcanzando un porcentaje de respuesta del $47 \%$ (117 estudiantes).

La muestra escogida para el análisis cualitativo de las memorias ha sido intencional, seleccionando aquellas centradas en los contenidos que mejor se ajustaban a los objetivos de la investigación. Se han analizado las memorias de cincuenta estudiantes. ${ }^{1}$

Tabla 2. Muestra del estudio. Estudiantes del Grado en Maestro en Educación Primaria e Infantil de la UCLM

\begin{tabular}{|l|r|}
\hline Cuestionario online & 117 \\
\hline Narraciones reflexivas & 50 \\
\hline Total & $\mathbf{1 6 7}$ \\
\hline
\end{tabular}

Fuente: Elaboración propia a partir de datos del estudio. 
En el diseño del cuestionario, se tomaron como referencia estudios previos (Capella et al., 2014; Campo, 2015; Folgueiras et al., 2013). Estaba compuesto por 21 preguntas, dirigidas a recoger información relativa a cuestiones organizativas, trabajo en grupo, implicación, relación con la asignatura y opinión sobre aspectos ligados a ella, junto a la percepción sobre sentimientos vividos y satisfacción con el proyecto ApS, utilizando una escala de valoración tipo Likert de 5 puntos (siendo 1 muy poco y 5 mucho).

En cuanto a la aportación cualitativa de las producciones escritas por el alumnado participante, el uso de las memorias o portafolios reflexivos, entre otras modalidades evaluativas, son técnicas habituales en investigaciones similares (Folgueiras, 2017; Loubet-Orozco, 2018). Se buscaba recabar percepciones y vivencias que complementasen el análisis cuantitativo y permitiesen profundizar en los aspectos de interés. Como veremos a continuación, en los testimonios seleccionados como apoyo a los resulta- dos del cuestionario, los estudiantes reflexionan sobre la experiencia personal vivida (individual y grupal), toman conciencia de las situaciones y contextos sociales observados e identifican los aprendizajes académicos (teórico-prácticos) movilizados en el marco de la asignatura Educación y Sociedad. Son, por tanto, tres ejes fundamentales que conjugan las percepciones de los estudiantes con el pensamiento teórico y con la práctica.

El análisis de los datos del cuestionario se realizó mediante el programa SPSS Statistics 24. Para el análisis cualitativo, tras el diseño de categorías, se utilizó el programa Atlas.ti 8.

\section{Resultados}

Una de las primeras cuestiones planteadas en este estudio fue en qué medida los y las estudiantes percibían la conexión entre el desarrollo del proyecto y los contenidos de la asignatura de Sociología de la Educación.

Figura 1. Valoración de la relación del ApS con la asignatura según el alumnado

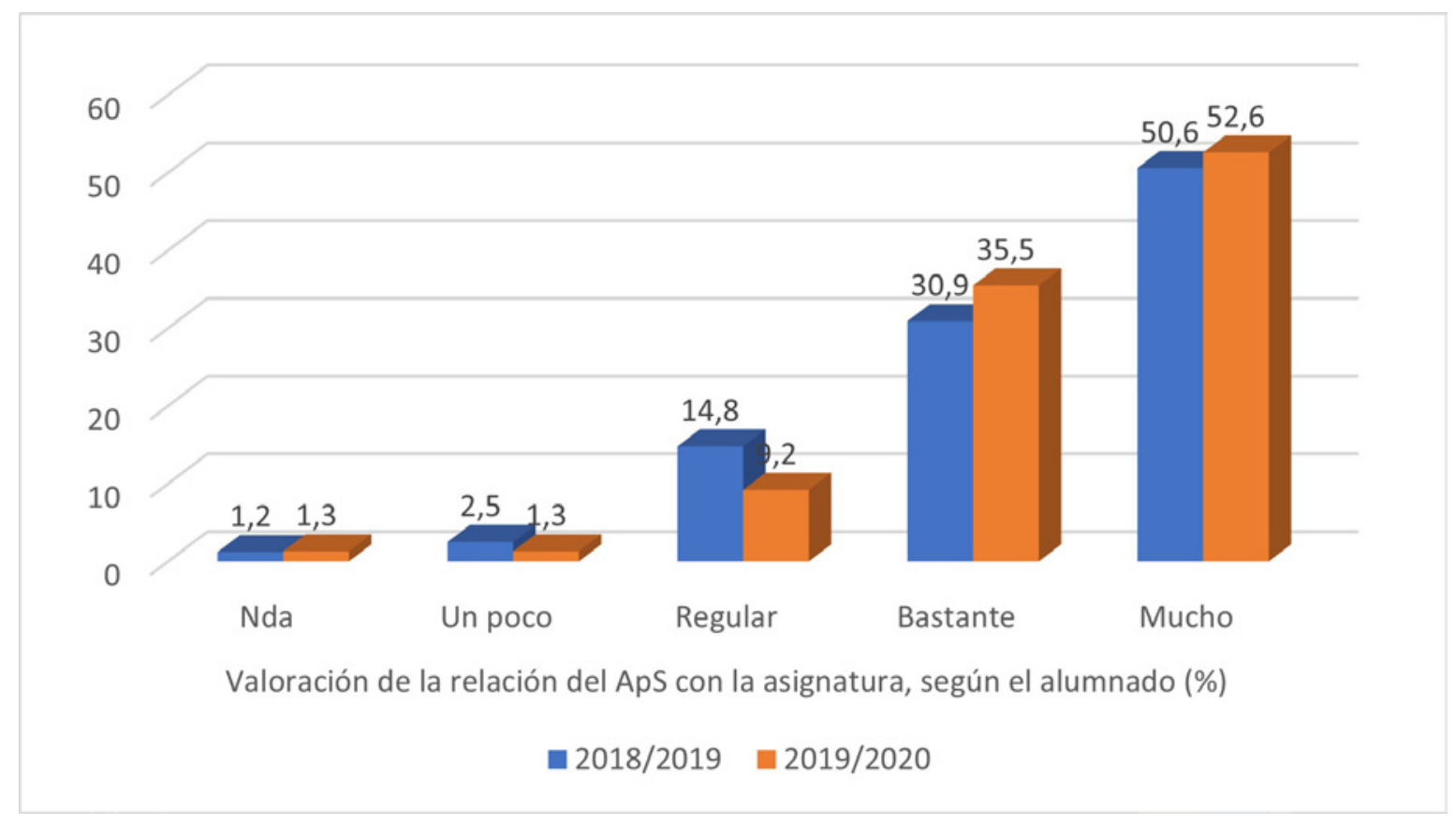

Fuente: Milla (2020, p. 18) 
La figura 1 nos muestra la elevada relación entre ambas percibidas por los estudiantes, situándose más del 50\% en la valoración más altas (5). La valoración media sobre este aspecto en el curso 2018/2019 es de 4,27 sobre 5 (DT: 0,895), incrementándose levemente en el curso 2019/2020 (media: 4,37; DT: 0,814).
Los estudiantes manifiestan que su experiencia ApS les ha ayudado a entender mejor los contenidos de la asignatura (media: 3,93; D.T.: $1,024) \mathrm{y}$, especialmente, a encontrar una mayor relación de la teoría con la práctica de la asignatura (media 4,14; D.T.: 0,989 ) y con la vida cotidiana (media 4,43; D.T.: 0,869).

\section{Figura 2. Relación del ApS con la teoría, con la vida cotidiana y con los contenidos. Curso 2019/2020}

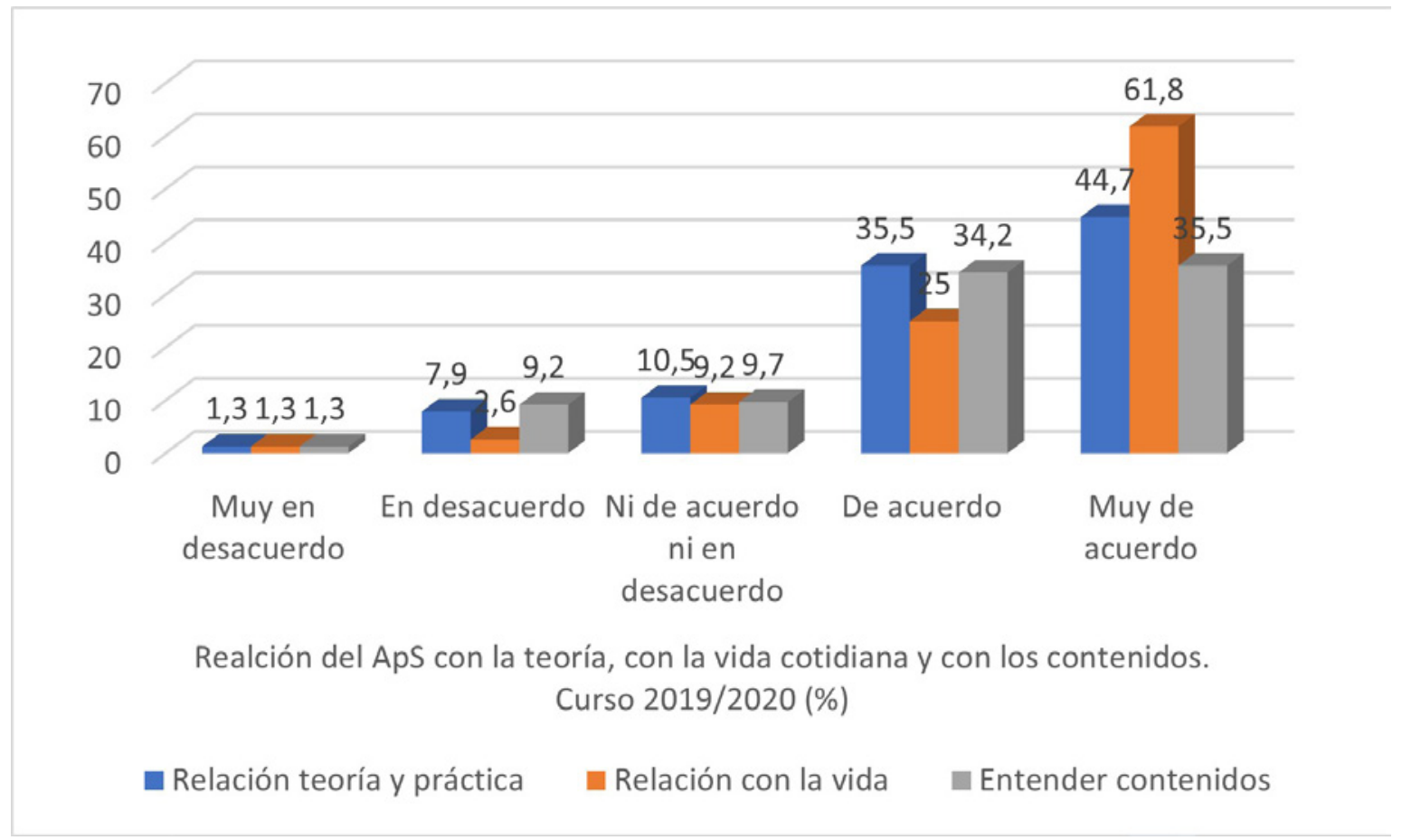

Fuente: Milla (2020, p. 18).

Del mismo modo, los resultados de la exploración cualitativa muestran la eficacia de la experiencia en la identificación y comprensión de los problemas sociológicos, a partir de la observación de situaciones reales en el contexto escolar. El encuentro con las desigualdades sociales, la diversidad cultural y la desigualdad de género, tiene un lugar destacado en las reflexiones de las estudiantes:

Hemos visto niños y familias con situaciones difíciles sociales y económicas, ya que hay un gran número de alumnado refugiado inmigrante. Esto me ha hecho ver la vida desde otra perspectiva, tenemos que ir a los retos para poder tratar las necesidades que se presentan y no acomodarnos, ya que todos y todas tienen derecho a ser educados. (M-4)

En el caso de la cultura gitana, la experiencia que he vivido en el ApS me ha sorprendido y tengo que reconocer que me ha servido para aclarar ciertos aspectos sobre su forma de vivir la vida, su educación y su cultura en general. La sociedad les pone reglas muy estrictas y como personas, debemos de ser capaces de priorizar el interior de nuestros iguales y dar oportunidad de conocer las vivencias de cada persona. (M-13) 
(...) haber trabajo con niños y niñas de etnia gitana, y ver que son como cualquier crío de su edad, con la misma imaginación, creatividad y capacidad de aprender. (M-18)

Observamos que el sexismo está muy presente en el aula, y es que las niñas no querían relacionarse con un niño que se les acercaba porque decían que era chico. Yo intenté mediar haciéndoles ver que no tenían por qué discriminarlo por el hecho de ser un chico, que todos eran iguales, independientemente del sexo. (M-22)

Observamos así, a partir de los testimonios recogidos, que las situaciones vivenciadas en la implementación de los proyectos de ApS han favorecido la práctica reflexiva como herramienta de trabajo docente en el aula. Cabe recordar que esta metodología se ha teorizado y difundido como modelo formativo de capacitación de los profesionales docentes (Domingo, 2013; LupiónCobos \& Gallego-García, 2017) y para el cambio educativo (Mayor \& Rodríguez, 2016).

La experiencia nos ha aportado conocimientos desde varias perspectivas para desarrollar nuestro pensamiento crítico, abstracto, reflexivo e innovador, para entender qué está ocurriendo dentro y fuera de la escuela. Nos ha servido para relacionar conceptos, aprender nuevas metodologías, taxonomías relativas a los procesos de aprendizaje y las habilidades cognitivas, la evaluación y las necesidades de cambio en el ámbito educativo... (M-9)

El patio es un espacio esencial en el contexto escolar y, especialmente, en la Educación Infantil. El tiempo de recreo es el momento en el que los niños y niñas disponen de más libertad para jugar, explorar, moverse y relacionarse con sus iguales. Esto lo hemos visto y lo hemos comprendido con la actividad de recreos activos del ApS. (M-27)

Otra muestra del valor del proyecto ApS en la formación del alumnado se muestran en las altas puntuaciones que arrojan las respuestas de los estudiantes, cuando son preguntados por la posibilidad de incluir esta metodología en otras asignaturas. Un $81,6 \%$ se manifiesta muy de acuerdo (media 4,75, D.T.: 0,635), ya que con esta experiencia han visto reforzada su vocación (73,7\% muy de acuerdo, media 4,58; D.T.: 0,868).

Gracias al desarrollo de la actividad ApS, los estudiantes perciben un desarrollo del propio conocimiento, conectando aprendizajes previos con nuevos contenidos (media 4,41; D.T.: 0,836 ), una mayor motivación hacia la asignatura $(76,3 \%$ de acuerdo o muy de acuerdo; media 4,04; D.T.: $0,916) \mathrm{y}$, en menor medida, un aumento de su atención (media 3,71; D.T.: 1,056).

Respecto al desarrollo de competencias vinculadas a la experiencia de ApS, las valoraciones son también altas, tanto las referidas a las competencias profesionales $(57,9 \%$ muy de acuerdo; media 4,37; D.T.: 0,877) como a las competencias transversales (53,9\% está muy de acuerdo, media 4,30 y D.T.: 0,924).

Casi el 54\% de los encuestados creen que no habrían aprendido más si el tiempo invertido en realizar ApS, se hubiese dedicado a dar clases en el aula.

Otros aspectos de gran relevancia son la valoración que realizan acerca de la contribución del ApS en la comprensión de su rol como futuros docentes (media 4,50; D.T.: 0,887) y la adquisición de conocimientos sobre cómo funciona un centro educativo (media 4,47; D.T.: 0,774).

En definitiva, se destaca la utilidad de la experiencia ApS en su formación como maestro/ maestra, otorgándole una valoración media de 4,71 sobre 5 (D.T.: 0,670 ) y que queda reflejada en estas palabras, expresadas por una estudiante:

(...) el papel del docente no se limita a impartir conocimientos a sus alumnas y alumnos, sino que va mucho más allá del ámbito educativo, ya que tiene que ejercer como guía y mediador en muchas ocasiones en situaciones conflictivas o complejas... no siempre en un contexto favorable. Es entonces cuando la escuela debe de actuar y proporcionarle al estudiante los medios que sean necesarios para facilitarle al máximo su situación y poner en práctica todas 
las técnicas y herramientas posibles para que la situación cambie a mejor. (M-4)

Dando un paso más, nos planteamos profundizar en las aportaciones del proyecto ApS a la adquisición de competencias profesionales concretas: las relativas al trabajo en equipo y al desarrollo de habilidades personales. Se observa que son aspectos muy bien valorados por la mayoría de los alumnos y que, además, muestran una correlación positiva con la satisfacción global con el proyecto ApS.

Cabe afirmar que parte del éxito en el desarrollo de estas actividades está relacionado con el buen funcionamiento del trabajo en grupo que destacan los alumnos (media 4,59, D.T.: $0,677)$ y que arroja una correlación significativa con la satisfacción expresada (bilateral al 0,$01 ; \mathrm{r}^{2}$ : $0,252)$. Las valoraciones volcadas en las memorias por las estudiantes protagonistas subrayan también la cooperación y el trabajo en equipo como una de las enseñanzas adquiridas:

Todos los días estuvimos trabajando de manera cooperativa en grupo. Eso provocó y facilitó que la propuesta que planteamos para el Aprendizaje y Servicio fuera más eficaz, dinámica, motivadora y productiva. (M-31)

Gracias al ApS ha sido una materia muy práctica y reflexiva, aprendiendo por nosotras mis- mas, desde nuestros errores, autoevaluarnos siendo críticas, además de aprender a trabajar en equipo y aumentar la creatividad. (M-11)

Como se viene afirmando, la evaluación muestra que la participación en estos proyectos favorece su formación en conocimientos necesarios para su futuro profesional. Tal y como destaca Uribe (2018), este es uno de los aspectos fundamentales que se derivan de la realización de actividades de este tipo. Concretamente, el ApS, beneficia la formación como docentes, relacionando su aprendizaje con la vida, desarrollando su propia competencia emocional (IbarrolaGarcía \& Artcuh, 2016; García \& Sánchez, 2017) y reforzando, en consecuencia, su vocación.

Parte de esos aprendizajes provienen del desarrollo de competencias vinculadas a sus habilidades personales. Preguntados los estudiantes por las percepciones emocionales en el desarrollo de su experiencia ApS, la mayoría manifiestan haberse sentido muy interesados, ilusionados, satisfechos, activos, entusiasmados, concentrados, despiertos, inspirados y fuertes.

En menor medida habían sentido nerviosismo y otorgan las puntuaciones medias más bajas a emociones "negativas", como irritabilidad, miedo, angustia, etc. La siguiente tabla muestra una ordenación de las valoraciones otorgadas a cada sentimiento.

Tabla 3. Valoración de los sentimientos en los participantes del ApS. Curso 2019/2020

\begin{tabular}{|c|c|c|}
\hline Ítem & Media (1-5) & D.T. \\
\hline Interesado & 4,78 & 0,479 \\
\hline Ilusionado & 4,7 & 0,589 \\
\hline Satisfecho & 4,57 & 0,806 \\
\hline Activo & 4,54 & 0,642 \\
\hline Entusiasmado & 4,47 & 0,856 \\
\hline Concentrado & 4,29 & 0,780 \\
\hline Despierto & 4,11 & 0,918 \\
\hline Decidido & 4,01 & 0,902 \\
\hline
\end{tabular}




\begin{tabular}{|c|c|c|}
\hline Ítem & Media (1-5) & D.T. \\
\hline Inspirado & 4 & 1,143 \\
\hline Fuerte & 3,93 & 0,971 \\
\hline Nervioso & 2,33 & 1,076 \\
\hline Afectado & 1,76 & 1,082 \\
\hline Agitado & 1,64 & 0,919 \\
\hline Asustado & 1,58 & 1,036 \\
\hline Angustiado & 1,46 & 0,807 \\
\hline Miedoso & 1,43 & 0,806 \\
\hline Avergonzado & 1,37 & 0,780 \\
\hline Culpable & 1,28 & 0,704 \\
\hline Irritable & 1,24 & 0,586 \\
\hline Agresivo & 1,12 & 0,431 \\
\hline
\end{tabular}

\section{Fuente: Elaboración propia a partir de datos del estudio.}

Cabe afirmar, por tanto, que esta experiencia resulta ser una fuente de sensaciones positivas, estimulantes y de desarrollo profesional y personal para los estudiantes del Grado en Maestro, nada más comenzar su andadura universitaria. Ese primer contacto con la realidad educativa en el comienzo de su vida universitaria supone para el alumnado una experiencia remarcable (Milla, 2020).

Todo ello lleva, como no podía ser de otro modo, a una elevada satisfacción general con el proyecto ApS. Un 60,5\% de los estudiantes en el curso 2028-19 otorgan la máxima puntuación a esta cuestión (media: 4,43; DT: 0,805), incrementándose hasta un 73,7\% en el curso 2019-20 (media 4,67; D.T.: 0,619).

En un análisis por género, no se han encontrado diferencias estadísticamente significativas en la satisfacción expresada (prueba de Kolmogorov-Smirnov: p: 439; tamaño del efecto: d Cohen: 0,18), si bien que son muchas las alumnas que hacen explícita esa satisfacción en sus discursos de "Educación y Sociedad" del Grado de Educación Infantil, donde todas las participantes son mujeres:
El APS me ha ayudado para conocerme a mí misma y saber qué cosas tengo que mejorar en mí a la hora de ser docente. (M-23)

Esta experiencia ha sido muy gratificante, no solamente hemos aprendido, sino que también hemos podido ayudar. (M-11)

(...) considero un enriquecimiento profesional esta experiencia y una gran satisfacción por ayudar (...). (M-4).

\section{Discusión y conclusiones}

Nuestros hallazgos de investigación han mostrado las posibilidades metodológicas del ApS en el ámbito de la formación inicial del profesorado, combinando aprendizaje de contenidos académicos con la prestación de un servicio a la comunidad. Pero el ApS es también una filosofía educativa que entronca con la dimensión transformadora de la educación que propugna toda la pedagogía crítica. En este sentido es donde hemos percibido el mayor calado de las aportaciones.

El análisis de las valoraciones de los y las estudiantes de magisterio revela la elevada satis- 
facción y la conexión de la experiencia formativa con las competencias curriculares y objetivos de aprendizaje de las asignaturas de Sociología. Observamos con ello la influencia positiva del ApS para reforzar y enriquecer el papel de la Sociología en la formación docente, un reto que perseguimos desde nuestra área de conocimiento y desde las Facultades de Educación.

En coincidencia con otros estudios que apuestan por extender el ApS en el ámbito universitario (Esparza et al., 2018), destacamos el impacto positivo de nuestra experiencia en los distintos colectivos implicados. Centrándonos en nuestros estudiantes, han aumentado su motivación, al contextualizar sus aprendizajes académicos y al haber podido utilizarlos para servir a la comunidad. Las clases de la Facultad se han hecho más dinámicas y participativas gracias a su implicación en el proyecto. Los colegios de infantil y primaria han podido cubrir algunas de sus necesidades, redundando en beneficio de los niños y niñas escolarizados. Por tanto, las Facultades de Educación han aumentado su impacto social en el entorno, contribuyendo al ejercicio de la responsabilidad social de la universidad.

La necesidad de promover sinergias entre todos los recursos educativos disponibles en la comunidad fue puesta de manifiesto en los informes internacionales sobre educación, como el de Coombs (1968), Faure (1973) o Delors (1996). En esta línea se han ido configurando diferentes propuestas educativas, como las Ciudades Educadoras, las Comunidades de Aprendizaje, los Planes Educativos de Entorno y las Redes Educativas Locales, el Proyecto Atlántida, etc. (Mayor-Paredes \& Rodríguez-Mar, 2015). El ApS viene a sumarse a esa lista, pero con la particularidad de permitir a los estudiantes universitarios ser verdaderos protagonistas.

Por último, cabe añadir la percepción de las profesoras del Área de Sociología que hemos liderado la implementación de los proyectos de ApS, coincidiendo en que la experiencia ha mejorado la difícil coordinación docente que existe en asignaturas compartidas (como Educación y Sociedad), sincronizando estilos y estrategias docentes que promueven el desarrollo de las competencias propuestas en el grado. En este sentido, los avances mostrados representan un avance significativo que habrá que investigar de manera sostenida en el futuro, preguntándonos si el ApS puede mejorar las estrategias didácticas, la motivación y evaluación del estudiantado universitario, estimulando la renovación y creatividad que necesita la enseñanza de la Sociología (Feito, 2020). Un desafío que contribuya a sentar las bases de una comunidad de aprendizaje con participación del profesorado y el alumnado.

\section{Nota}

1 Los fragmentos que se incluyen a lo largo del texto se identifican con un código (M-1 a M-50) correspondiente al número asignado a cada memoria reflexiva. Las autoras de estas memorias son estudiantes de $2^{\circ}$ curso del Grado de Maestro de Educación Infantil del Campus de Ciudad Real y responden a un perfil sociodemográfico relativamente homogéneo: mujeres jóvenes, entre 19 y 26 años, que viven en Ciudad Real o su entorno geográfico más próximo.

\section{Referencias bibliográficas}

Álvarez-Castillo, J.L., Martínez-Usarralde, M.J., González-González, H., \& BuenestadoFernández, M. (2017). El aprendizaje-servicio en la formación del profesorado de las universidades españolas. Revista Española de Pedagogía, 75(267), 199-217. http://doi.org/10.22550/REP75-2-2017-02

Brozmanová, A., Heinzová, Z., \& Chovancová, K. (2016). The Impact of Service-Learning on Students' Key Competences. The International Journal of Research on ServiceLearning and Community Engagement, 4(1), 367-276. https://bit.ly/3gDcFV1

Campo, L. (2015). Evaluar para mejorar los proyectos de aprendizaje servicio en la universidad. RIDAS, Revista Iberoamericana de Aprendizaje y Servicio, 1, 91-111. http://doi.org/10.1344/RIDAS2015.1.6

Capella, C., Gil, J., \& Martí, M. (2014). La metodología del Aprendizaje-Servicio en la Educación Física. Apunts. Educación Física y Deportes, 116(2), 33-43. 
https://doi.org/10.5672/apunts.2014-0983.es.

Coombs, P.H. (1968). La crisis mundial de la educación. Península.

Delors, J. (1996). La educación encierra un tesoro. Santillana.

Domingo, A. (2013). Práctica reflexiva para docentes. De la reflexión ocasional a la reflexión metodológica. Publicia.

Esparza, M., Morín, V., \& Rubio, L. (2018). La incorporación del aprendizaje-servicio en la universidad: La experiencia de la Universidad de Barcelona. RIDAS, Revista Iberoamericana de Aprendizaje Servicio, 6, 103-114. https://doi.org/10.1344/RIDAS2018.6.10

Faure, E. (1973). Aprender a ser. La educación del futuro. Alianza Editorial.

Feito, R. (2020). La difícil coordinación en la enseñanza de la Sociología. Revista Española de Sociología, 29(2), 401-409. https://doi.org/10.22325/fes/res.2020.23

Ferrán-Zubillaga, A., \& Guinot-Viciano, C. (2012). Aprendizaje-servicio: propuesta metodológica para trabajar competencias. Portularia, (12), 187-195.

http://doi.org/10.5218/prts.2012.0020

Folgueiras, P. (2017). Evaluación y actividades de aprendizaje en los proyectos de aprendizajeservicio. En L. Rubio y A. Escofet (Coords.), Aprendizaje-Servicio (ApS): Claves para su desarrollo en la Universidad (pp. 97-110). Octaedro.

Folgueiras, P., Luna, E., \& Puig, G. (2013). Service learning: Study of the degree of satisfaction of university students. Revista de Educación, (362), 159-185.

http://doi.org/10.4438/1988-592X-RE-2011-362-157

Furco, A. (2004). Impacto de los proyectos de aprendizaje-servicio. En Aprendizaje y servicio solidario en la Educación Superior y en los sistemas educativos latinoamericanos. Actas del $7 \mathrm{mo}$. Seminario Internacional "Aprendizaje y Servicio Solidario". Buenos Aires, 6 y 7 de octubre de 2004.

García-García M., \& Sánchez-Calleja, L. (2017). El aprendizaje servicio y el desarrollo de las competencias emocionales en la formación inicial del profesorado. Contextos Educativos. Revista de Educación, 20, 127-145. https://doi.org/10.18172/con.2991
Guerrero-Serón, A. (2007). La doble contribución de la sociología a la formación del profesorado, Revista Internacional de Sociología, 65(48), 203-220. https://doi.org/10.3989/ris.2007.i48.74

Gutiérrez, M., \& Moreno, P. (2018). El aprendizaje servicio como metodología para la formación integral de los estudiantes universitarios. Edetania. Estudios y propuestas socioeducativas, (53), 185-202. https://bit.ly/3b5NedG

Ibarrola-García, S., \& Artuch, R. (2016). La docencia en la universidad y el compromiso social y educativo. Contextos educativos: Revista de educación 19, 105-120. https://doi.org/10.18172/con.2763

Kaplan, C.V. (2012). Mirada social, exclusión simbólica y autoestigmación: Experiencias subjetivas de jóvenes de educación secundaria. En C.V. Kaplan, L. Krotsch y V. Orce, Con ojos de joven: Relaciones entre desigualdad, violencia y condición estudiantil (pp.15-78). Facultad de Filosofía y Letras de la Universidad de Buenos Aires.

López-Gamboa, G.E. (2019). Representaciones sociales de estudiantes de Magisterio acerca del éxito y el fracaso escolar: Una investigación en las escuelas normales del Estado de Yucatán. (Tesis doctoral). Universidad de Castilla-La Mancha. https://bit.ly/3hEX9ck

Loubet-Orozco L. (2018): Contribución de la sociología en la formación docente. Un ejercicio de intervención en México. Revista de Sociología de la Educación (RASE), 11(1), 5-20. https://doi.org/10.7203/RASE.11.1.10598

Lupión-Cobos, T., \& Gallego-García, M.M. (2017). Compartiendo la mirada: una experiencia en práctica flexiva para formación permanente. Revista Electrónica Interuniversitaria de Formación del Profesorado, 20(1), 127-144. http://doi.org/10.6018/reifop/20.1.244931

Mayor-Paredes, D., \& Rodríguez-Mar, D. (2015). Aprendizaje-Servicio: Construyendo espacios de intersección entre la escuela-comunidad-universidad. Profesorado. Revista de currículum y formación del profesorado, 19(1), 262-279. https://bit.ly/2EBGGY9

Mayor-Paredes, D., \& Rodríguez-Mar, D. (2016). Aprendizaje-servicio y práctica docente: una relación para el cambio educativo. Revista 
de Investigación Educativa, 34(2), 535-552. https://doi.org/10.6018/rie.34.2.231401

Milla-Martínez, L. (2020). El Aprendizaje-Servicio en la formación de futuros docentes: Una experiencia en el grado de Educación Primaria de la Facultad de Educación de Albacete. (Trabajo Fin de Master Universitario de Investigación e Innovación Educativa). Universidad de Castilla-La Mancha. https://bit.ly/32wcaqJ

Mills, C.W. [1959] (1986). La imaginación sociológica. Fondo de Cultura Económica.

Obiol i Francés, S., Beltrán-Llavador, J., Box-Varela, Z., Gabaldón-Estevan, D., Hernàndez-Dobon F., Martínez-Morales, I., \& Martínez-Morales, J.R. (2019). Enseñar Sociología a quienes no estudian Sociología, 1085-1095. V Congreso de Innovación Educativa y Docencia en Red. Universidad Pública de Valencia.

http://dx.doi.org/10.4995/INRED2019.2019.10476

Pérez-Gómez, A.I. (1993). La formación del docente como intelectual comprometido. La tarea de provocar la reconstrucción del conocimiento, Signos. Teoría y práctica de la educación, 8/9, 42-53. https://bit.ly/34HDtBc

Pérez-Gómez, A.I (2010). Aprender a educar. Nuevos desafíos para la formación de docentes. Revista Interuniversitaria de Formación del Profesorado, 68(24,2), 37-60. https://bit.ly/3lkcvFs

Perrenoud, Ph. (2002). La place de la sociologie dans la formation des enseignants: réflexions didactiques. Education et Sociétés, 1/9, 87- 99. https://doi.org/10.3917/es.009.0087
Rubio, L., \& Escofet, A. (Coords.) (2017). AprendizajeServicio (ApS): Claves para su desarrollo en la Universidad. Octaedro.

San Román, S., Vecina, C., Usategui, E., del Valle, A.I., \& Venegas, M. (2015). Representaciones sociales y orientación educativa del profesorado. Education Policy Analysis Archives/ Archivos Analíticos de Políticas Educativas, 23, 1-18. https://doi.org/10.14507/epaa.v23.2088

Tapia, M.N. (2008). Calidad académica y responsabilidad social: el aprendizaje servicio como puente entre dos culturas universitarias. En M. Martínez (Ed.), Aprendizaje servicio y responsabilidad social de las universidades. Octaedro-ICE.

Tapia, M.N. (2014). La aportación del aprendizaje-servicio en el mundo. Cuadernos de Pedagogía, 450, 54-56. https://bit.ly/2G57f8G

Tarabini, A. (2015). La meritocracia en la mente del profesorado: un análisis de los discursos docentes en relación al éxito, fracaso y abandono escolar. Revista de la Asociación de Sociología de la Educación, 8(3), 349-360. https://bit.ly/2Qz3BWp

Uribe, A. (2018). Percepción de los estudiantes de educación inicial frente al desarrollo de experiencias formativas en modalidad $\mathrm{A}+\mathrm{S}$. Revista Electrónica de Investigación Educativa, 20(4), 110-122. http://doi.org/10.24320/redie.2018.20.4.1826

Venegas, M. (2012). Sociología y formación del profesorado: aportaciones y balance en el nuevo paradigma universitario. Educatio Siglo XXI, 30(2), 403-422. https://bit.ly/2DbKdfi 